\title{
Effect of Cesarean Section on Demand on the phenomena of Increasing Cesarean Section Rate at Women's Health Hospital
}

Sara A. Ibrahim ${ }^{1}$, Manal F. Mostafa ${ }^{2}$ \& Entisar M. Youness ${ }^{3}$.

1. Nursing Specialist at Women's Health Hospital, Assiut University, Egypt.

2. Professor of Obstetrics \& Gynecological Nursing, Faculty of Nursing- Assiut University, Egypt.

3. Assistant Professor of Obstetrics and Gynecology Faculty of Nursing, Assiut University, Egypt.

\begin{abstract}
Aims of the study were to determine the hospital based rate of C.S at Assiut Women's Health Hospital and assess the effect of C.S on demand on increasing C.S rate. Setting: This study was conducted at Assiut Women's Health Hospital, postpartum unit .Sample: A convenience sample (1500) of all postpartum women who had C.S during a three months period. Design: A cross -sectional descriptive study design was used in this study. Results: Cesarean section rate in hospital was $47.3 \%$ at 2016 and cesarean section on demand accounts for about $5.5 \%$ of the rate of C.S and in the present study findings. Conclusion: C.S which was done on maternal demand was accounted for $5.5 \%$, as it might increase the rate of hospital based rate of C.S. Recommendations: Implementation of counseling sessions for pregnant women to improve awareness of vaginal delivery benefits, indications for C.S and the consequences of performing an elective or an unnecessary C.S.
\end{abstract}

Key words: C.S \& Cesarean Section On Demand.

\section{Introduction}

Cesarean birth is the delivery of a baby through incisions made in the mother's abdomen and uterus. (ACOG, 2015) Cesarean section is the most commonly performed operation in Obstetrics. It had saved uncountable lives of women and their babies. In last few years the rates of this operation are increasing (Al Ameen, 2017) Caesarean section may be necessary when vaginal delivery might pose a risk to the mother or baby - for example due to prolonged labour, foetal distress, or because the baby is presenting in an abnormal position. However, caesarean sections can cause significant complications, disability or death, particularly in settings that lack the facilities to conduct safe surgeries or treat potential complications (WHO, 2015) A study that was done in the Arab region in 2009 has reported an increasing trend of cesarean section delivery in this region. There are very few studies in Egypt that examined hospital based CS rates and indications in Egypt (Khawaga, 2009).

The study found that the CS rate in Egypt was 26\% in 2003. The study also found that the discrepancies in CS rate by rural and urban residence were relatively small. The 2008 Demographic and Health Survey in Egypt also showed that the CS rate in the five years preceding the survey was about $25 \%$ of deliveries nationwide and $37 \%$ of deliveries in urban areas (El-zanaty, 2008).

The Cesarean section rate at Assiut Women's Health Hospital was 32\% in 2008 and 38\% in 2011 and 47.2 in 2014. The most common indication in both time intervals was a previous cesarean delivery. The second and third most common indications were cephalopelvic disproportion, dystocia and failure of progress and other obstetric complications. The fourth most common indication was preeclampsia/eclampsia in 2008 and fetal distress in 2011.The number of women who underwent a cesarean delivery at their own request increased from 5.9\% in 2008 to $11.5 \%$ in 2011 (Abdel-Aleem, 2013).

A cesarean section is performed for maternal indications, fetal indications or both. The leading indications for cesarean section are previous cesarean section, breech presentation, dystocia and fetal distress. These indications responsible for $85 \%$ of all cesarean sections. For certain clinical conditions such as placenta previa or uterine rupture; cesarean section is firmly established as the safest mode of delivery (Joy, 2014).

The proportion of caesarean sections at the population level is a measure of the level of access to and use of this intervention. It can serve as a guideline for policy-makers and governments in assessing progress in maternal and infant health and in monitoring emergency obstetric care and resource use (WHO, 2015).

Current Institute for Health and Clinical Excellence guidelines state that pregnant women should be offered evidence-based information to support decision-making on the method of childbirth during the ante-natal period. This should include information about the risks and benefits of caesarean section, the situations when caesarean sections are recommended, such as presumed fetal compromise, failure to progress in labour and breech presentation, what the procedure involves, and the implications for 
future pregnancies and birth after caesarean section (RCOG, 2015).

However, in line with NICE guidance in the UK, RCOG also believe that if a woman requests a caesarean section, she should be informed of all the risks and benefits of the procedure and, if appropriate, be given the support of a counselor or psychologist. If she remains certain that caesarean section is the right option for her, then that choice should be fully respected (Rcog, 2015).

Cesarean section on maternal request (or "on demand") refers to a primary cesarean delivery performed because the mother requests this method of delivery in the absence of standard medical/obstetrical indications for avoiding vaginal birth. A woman's right to be actively involved in choosing the route of her delivery is now widely accepted by clinicians and patients (ACOG, 2013).

The incidence of cesarean section on maternal request and its contribution to the overall increase in the cesarean section rate are not well known, but it is estimated that $2.5 \%$ of all births in the United States are cesarean section on maternal request (NIH, 2012).

\section{Significance of the study}

An increase in cesarean section birth rate is evident worldwide, since this trend is rapidly gaining epidemic status with unpredictable consequences regarding the reproductive and overall women's health, there is a need for systematic collection and analysis of cesarean section occurrence data.

The effect of nursing role in cesarean section on demand on increasing cesarean section rate is important; therefore, a study examining the decision - making process will increase awareness of the factors that contribute to a women's decision for a cesarean birth (Denise, 2013).

\section{Aims of the study}

This study aims to

1. Determine the hospital based rate of cesarean section at Assiut Women's Health Hospital.

2. Assess the effect of cesarean section on demand on increasing cesarean section rate.

\section{Patients \& Methods \\ I-Technical design \\ Research Design}

A cross -sectional descriptive study design was used in this study.

Setting

This study was conducted at Assiut Women's Health Hospital.

\section{Patient}

The study included all postpartum women who had a cesarean section to examine women's role in the decision to perform C.S.

Sample

A convenience sample of all post-partum women who had cesarean birth during a three months period of time from 1-5- 2016 to 31-7- 2016.They were 1500 women that included in this study.

Research question

Is the cesarean section on demand leads to increase the rate of cesarean section?

Inclusion criteria

- Women who delivered by cesarean section.

- Women who accepted to participate.

Tool of the Study

A semi-structured interviewing questionnaire was used to collect the study data. Data were collected by using the Cesarean Birth Decision Survey, an interview questionnaire developed by Denise, (2013). And was taken as it is with the previous validity and reliability of the tool. The questionnaire consisted of personal data and questions that addressed problems with the pregnancy, reasons for the cesarean surgery, and what information was provided and by whom.

1- The personal data included the following

Age, educational background, residence and employment.

2- Data related to place of prenatal care providers included the following

Private clinic or $\mathrm{MCH}$ center

3- Data related to antenatal risk factors which included the following

Breech presentation, preterm labor, preeclampsia, large baby, small baby, twins, triplets, diabetes and bleeding.

4- Data related to delivery history which included the following

Number of deliveries and number of cesarean births.

5- Data related to indications for this cesarean birth which included the following:

- Maternal indications, Indications related to labor as failure of induction of labor, non-medical indications (on demand).

6- Data related to sources of information woman use in making her decision to be cesarean section on demand which included the following

Doctor, traditional birth attendant, nurse, movies, internet, television, friends, relatives, books or personal experience.

7- Data related to what information women remember being provided to her prior to cesarean section which included the following:

Risks of surgery to her, risks of surgery to the fetus. Types of anesthesia used during recovery, length of 
procedure, what to expect while in the operating room, what to expect while in the recovery room, types of pain relief drugs used during recovery and what to expect about recovery.

Short-answer questions addressed additional comments as well as how the participant ranked the importance of reasons for the cesarean and information provided to the women that help them to decide her cesarean section on demand.

Additional statements to assess women satisfaction about cesarean section as

Women understand information provided to her well. Women had the opportunity to ask questions.

Women feel the information provided to her was helpful.

Women received all additional information she need. Women felt comfort to the decision she took.

Women were not distressed or anxious about cesarean section.

Women felt well supported by staff during her decision to have

cesarean section.

The staff communicated well with them during their decision to have

cesarean section.

-Based on likert scale (agree-agree to some extentnot agree) satisfaction level was determined and the scoring system was

Level of satisfaction Score

Satisfied $>60 \%$ of the statements

Not satisfied $<60 \%$ of the statements

\section{II-Administrative design:}

An official permission was obtained from the director of Women's Health Hospital Assiut University to proceed with this study as well as an Ethical Committee of Faculty of Nursing Assiut University.

\section{Ethical consideration}

1-Oral consent was taken from women because they were in pain and most of them were illiterate.

2-Research proposal was approved from Ethical Research Committee of Faculty of nursing, Assiut University.

3-The study was followed common Ethical principles in clinical research.

4-There was no risks for study subjects during application of the research.

5 -The women were assumed that all information obtained would be confidential and would be used only for the purpose of study.

6-Women had the right to refuse to participate or withdraw from the study without any rational.

7- Privacy of women was considered during collection of data.

\section{III- Operational design}

This design involved description of preparatory phase, pilot study and procedure.

\section{Preparatory phase}

The investigator reviewed related literature of national and international text books, articles and scientific magazines. The tool was then prepared. Pilot study

A pilot study was carried out on $10 \%$ of the study sample (150 women, all women who had cesarean section during a period of 9 days) to test the content, validity, feasibility, clarity and objectivity of the study tool. Minor modifications were done to meet the aim of the study.

Women who included in the pilot study were also included in the current study.

Procedure

- All women who delivered during the determined period were included in the study.

- Women were interviewed at the postpartum ward every day during the determined period at Assiut Women's Health Hospital within 24 hours postpartum.

- At the beginning of each interview, the investigator greeted the woman and introduced herself to the woman.

- Then each woman was interviewed separately to obtain personal data, obstetric history, antenatal risk factors and indications for this cesarean section then the questionnaire items were filled by the researcher.

- Each interview took about 15-20 minutes to fill the entire questionnaire

- All results were anonymous and the women were not contacted in the future.

- At the end of the study, the researcher calculated the rate of normal vaginal deliveries to determine the rate of cesarean section through the maternity records of the registration office during the determined study period

\section{Statistical Analysis}

Data was coded to allow for data entry. Analysis was performed using Statistical Package for the Social Science (SPSS version 20). Descriptive statistics were used to illustrate the participants' characteristics. The frequencies and percentages of the categorical variables were displayed. Chi-square test was used to determine p.value was demonstrated in the form of tables. 


\section{Results}

Table(1): Distribution of the studied sample according to indications for C.S.

\begin{tabular}{|l|c|c|}
\hline \multicolumn{1}{|c|}{ Indications for C.S: } & No.(1500) & $\%$ \\
\hline On demand (subjective indications) & $\mathbf{8 3}$ & $\mathbf{5 . 5}$ \\
\hline Fetal indications: & 130 & 8.7 \\
\hline Twins/triplets & 121 & 8.1 \\
\hline Breech presentation & 113 & 7.5 \\
\hline Problem with the baby & 76 & 5.1 \\
\hline Preterm labor & 24 & 1.6 \\
\hline Large baby ( macrosomia) & & 16.2 \\
\hline Maternal indications: & 243 & 5.7 \\
\hline Preeclampsia & 86 & 2.7 \\
\hline Bleeding & 40 & 3.2 \\
\hline Diabetes & & 5.1 \\
\hline Indications related to labor: & 48 & 9.7 \\
\hline Failure of induction of labor & 77 & 24.7 \\
\hline Failure of progress of labor & 146 & 16.5 \\
\hline Attempt to have VBAC did not work & 371 & 3.1 \\
\hline Emergency & 248 & 2.1 \\
\hline Previous C.S. in labor & 47 & 1.8 \\
\hline Tender scar & 32 & 0.6 \\
\hline Bleeding & 27 & 0.3 \\
\hline - Fetal distress & 9 & 0.3 \\
\hline Cord prolapse & 4 & 60.8 \\
\hline - Eclampsia & 4 & 22.3 \\
\hline - Footling presentation & 912 & 15.1 \\
\hline Previous cesarean birth & & 7.4 \\
\hline Non-medical indications: & 334 & 5.3 \\
\hline Safer for the baby & 226 & 2.4 \\
\hline Doctor recommendation & 171 & \\
\hline Convenience of scheduling birth & 108 & \\
\hline Prior traumatic birth experience & 79 & \\
\hline Fear of labor pain & 36 & \\
\hline Fear of loss of control in labor & & \\
\hline
\end{tabular}

\#more than one answer was checked.

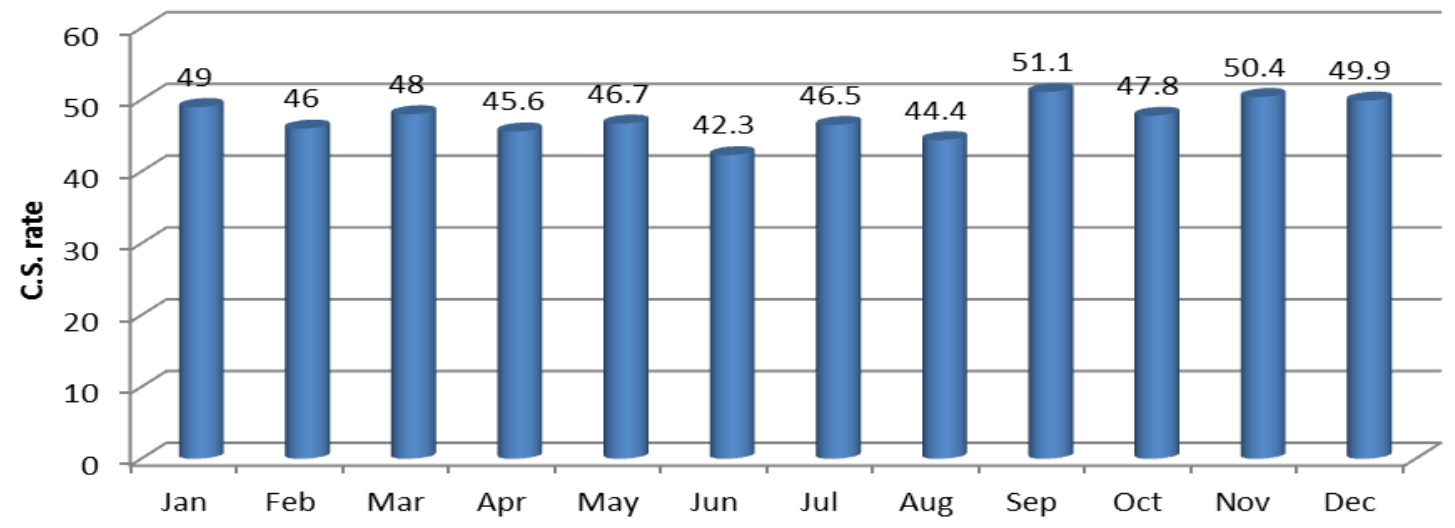

Fig(1): Rate of cesarean section 2016 per month. 
Table (1): Shows that $5.5 \%$ of studied sample had cesarean section on demand. Fetal indications for cesarean section included twins pregnancy that represent $8.7 \%$. Maternal indications for cesarean section include preeclampsia that represents $16.2 \%$. Indications that related to labor show that $60.8 \%$ of studied sample having C .S due to previous cesarean delivery. Non-medical indications represent that $22.3 \%$ of women having cesarean section as it is safer for the baby.

Fig (1): Shows the rate of cesarean section at 2016 per month, the highest rate was in September 51.1\% and the lowest rate was in June $42.3 \%$.

\section{Discussion}

Aims of the study were to determine the hospital based rate of C.S at Assiut Women's Health Hospital and assess the effect of C.S on demand on increasing C.S rate. As regards distribution of the studied women's according to indications for C.S the present study findings revealed that more than half of them had previous cesarean birth, nearly half of them were emergency, less than half of them had cesarean section as it is safer for the baby. The results which were carried out by Dawood, (2017) is consistent with present study findings that Previous CS was the most important indication for CS. In the past three years to be $58.1 \%$ in $2013,52.3 \%$ in 2014 and reach $52.9 \%$ in 2015 , and the other indications make little contribution for indication of cesarean section, the second larger contribution for indication of cesarean section in 2013 was cephalopelvic disproportion $9.4 \%$, in 2014 was failed trial 9\%, in 2015 was failed trial $8.9 \%$.

The findings of El Khyat et al., (20013), were similar to the present study findings that at Cairo University Repeat cesarean section (RCS) was the main indication and a large proportion of patients were having previous one cesarean section with an incidence of about $43.5 \%$ in the past 5 years.

The results of the present study came in line with Santhanalakshmi et al., (2015) who analyzed indications of cesarean section over a period of 4 years at the Department of Obstetrics and Gynecology Karpaga Vinayaga Institute of Medical sciences and Research center, Maduranthagam, Tamil Nadu, India where the incidence of cesarean section was $12.5 \%$. The commonest indication for CS was repeat cesarean (43\%) followed by CPD (15\%).

In contrary to the present study findings, the study which carried out by Sercekus \& Okumus, (2009) found that the most common source of fear related to childbirth was reported to be labour pain, and the participants in their study reported to be afraid of failure to give birth and losing their control, another study carried out by Buyukbayrak, (2010) in Turkey stated that majority of women preferred CS due to fear of VD, a fifth of the participants was reported to prefer CS due to finding it safer for babies. Also in a study in Singapore Chong, (2003) it was also found that more than half of the women preferred CS because of avoidance of labour and stress.

Also in contrary to present study findings, the study of Denise, (2013) found that the main reason cited for the cesarean surgeries was the women's perception of doctor recommendation; however, because the doctors were not questioned, it is unclear what the physicians may have considered to be the primary reason.

The rational, these differences in indications of cesarean section may be contributing to policy of Assiut university hospital, obstetric history of women as most of them had previous section and requiring emergency cesarean section.

The present study indicates that cesarean surgeries on maternal request account for a very small percentage of the overall cesarean section. The findings of Robson, (2009) which is consistent with the present study findings that maternal request for cesarean sections in Australia in 2006, representing at least 17 percent of all elective cesarean sections, and slightly more than 3 percent of all births. In contrary to the present study findings, a study conducted by The American College of Obstetricians \& Gynecologists, (2007) on 600 obstetricians and gynecologists reported that more than half of the participants $(53 \%)$ performed C.S on maternal request.

The rational, cesarean surgeries on maternal request account for a very small percentage of the overall cesarean surgery rate because of the policy of hospital of current study.

As regard distribution of the studied women according to their satisfaction about decision of cesarean section the present study findings revealed that most of them understand information provided, had the opportunity to ask questions, information provided was helpful to them and they were satisfied with decision to have a cesarean birth. The study of Denise, (2013) is consistent with the present study findings, that most of the women felt they understood the information that was provided and felt they had the opportunity to ask questions. Overall the vast majority of study participants felt the information provided was helpful.

Cesarean section on demand accounts for about $5.5 \%$ of the rate of cesarean section in the present study findings showing that in the public sector the wishes of pregnant women are subordinate to work routines and policy of hospital which limits the their autonomy. The study of Panda, (2013) is consistent 
with the present study findings, the rate of Cesarean delivery on maternal request (CDMR) is $6.2 \%$ (in year 2008-2010), while recent national audit in UK revealed that $7 \%$ all elective cesarean session were performed precisely for this reason.

The rational, Cesarean section on demand accounts for about $5.5 \%$ of the rate of cesarean section at Assiut university hospital as the wishes of pregnant women were subordinate to work routines and policy of hospital which limits the their autonomy.

\section{Conclusion}

Based on the findings of the present study, was concluded that cesarean sections which was done on maternal demand was accounted for $5.5 \%$, as it might increase the rate of hospital based rate of cesarean section.

\section{Recommendations}

Based on the findings of the current study it was recommended that

1. Provide counseling for pregnant women during the antenatal period about modes of delivery, their indications, advantages and adverse consequences which will enable them to make an informed decision.

2. Prenatal preparatory educational sessions for pregnant mothers to increase awareness of vaginal delivery benefits, the indications for cesarean section and the consequences of performing an elective or an unnecessary cesarean section.

\section{References}

1. Abdel Aleem H., Shabban M., \& Hassanin I., (2013): Analysis of cesarean delivery at Assiut University Hospital using Ten Group Classification System ,International Journal of Gynecology and Obstetrics,(123 ) 119-123.

2. American College of Obstetricians \& Gynecologists and Society for MaternalFetal Medicine (2014): Safe Prevention of the Primary Cesarean Delivery.

3. American College of Obstetricians \& Gynecologists (2013): ACOG committee opinion no. 559: Cesarean delivery on maternal request. Obstet Gynecol ; 121:904.

4. Acog (2011): Practice Bulletin No. 120: Use of prophylactic antibiotics in labor and delivery. Obstet Gynecol. 117(6):1472-83.

5. Acog (2007): Committee Opinion No. 386 November: cesarean delivery on maternal request. Obstet Gynecol. 2007; 110(5):1209-12.

6. Buyukbayrak E., (2010): Caesarean delivery or vaginal birth: preference of Turkish pregnant women and influencing factors. $\mathrm{J}$ Obstet Gynaecol; 30(2):155-8.
7. Chong E., Mongelli M., (2013): Attitudes of Singapore women toward cesarean and vaginal deliveries. International journal of gynaecology and obstetrics: the official organ of the International Federation of Gynaecology and Obstetrics. 80(2):189-94.

8. Dawood A., El-shwaikh L., (2017): A Three Year Retrospective Study of Caesarean Section Rate at Tanta University Hospitals. Journal of Gynecology and Obstetrics. Vol. 5, No. 2, , pp. 25-30.doi:10.11648/j.jgo.20170502.11

9. Denise, M., Puia (2013): The Cesarean Decision Survey. The Journal of Perinatal Education, 22(4), 212-225. http://doi.org/10.1891/1058-1243.22.4.212.

10. El-Khayat W., (2013): Cesarean Section Rate at a Tertiary University Hospital in Egypt in Five Years Period (2008 -2012). KAJOG; Kasr Al-Aini Journal Of Obstetrics \& Gynecology. 4 (3). 66-74.

11. El-Zanaty. F., \& Ann W., (2015): Egypt Demographic and Health Survey 2008.Cairo, Egypt: Ministry of Health. 8-Josipovic B,Stojkanovic D,Brkovic I,Analysis of CS delivery according to Robson Classification,Original scientific paper,(39)1:145-150.

12. Joy. S., (2014): Cesarean Delivery, Medscape, Vol (4), No (3).

13. Khawaga. M., (2009): Hospital based cesarean section in the Arab region: an overview. East Mediterr Health J; 15 (2):458-69.

14. NIH State-of-the-Science Conference, (2012): Statement on cesarean delivery on maternal request.NIH Consensus state Sci Statements; 23:1-29 Available at: http:// consensus.nih.gov/2006/cesarean statem

15. Panda S., (2013): Review of Cesarean Section on Maternal Request in A Tertiary care Institute ; Scenario in Developing Country. Kathmandu Univ Med J ;44(4):349-354.

16. Rcog, (2015): RCOG statement on new World Health Organization (WHO) guidance on caesarean section.

17. Robson S., (2009): Estimating the rate of cesarean section by maternal request: anonymous survey of obstetricians in Australia.

18. Santhanalakshmi C., (2015): Retrospective Analysis of Cesarean Section in a Tertiary Care Hospital, international Journal of Science and Research; 4, 9-12) .

19. Sercekus P., Okumus H., (2009): Fears associated with childbirth among nulliparous women in Turkey. Midwifery; 25(2):155-62.

20. WHO, (2015): Statement on Caesarean Section Rates. 